\title{
Homing and in situ differentiation of resident pulmonary lymphocytes
}

\author{
Gek-Kee Sim, Ramanujam Rajaserkar', Mark Dessing and Andrel Augustin ${ }^{1,2}$ \\ Basel Institute for Immunology, Grenzacherstrasse 487, CH-4005 Basel, Switzerland \\ 'Department of Medicine, National Jewish Center for Immunology and Respiratory Medicine, 1400, \\ Jackson Street, Denver, CO 80206, USA \\ 2Department of Immunology, University of Colorado Health Sciences Center, Denver, CO 80262, USA
}

Key words: $\gamma^{\delta}$ T cells, extrathymic differentiation, lymphoid precursors, RAG-1, RAG-2, resident pulmonary lymphocytes, scid reconstitution, selection

\begin{abstract}
At birth, T lymphocytes which colonize the lung are malnly of the $\gamma \delta$ subset, while $\alpha \beta \mathrm{T}$ cells predominate in the spleen. Thus, the lung 18 a preferred site for the homing of $\gamma \delta \mathrm{T}$ cells in the perinatal perlod. However, after blrth, the pattern of $V_{\gamma}$ gene usage among resident pulmonary lymphocytes (RPL) changes with age, from a predominance of $V_{7} 6$ at birth to a predominance of $V_{\gamma} 4$ in older mice. The generation of the $V_{\gamma} 6$ fraction appears to be thymus dependent, since in athymic nude mice, the $V_{\gamma} 6$ population present at birth is replaced by $V_{\gamma} 4 T$ cells. In the postnatal period, both RAG-1 and RAG-2 genes are expreseed at high levels In the RPL populatlon. TCR bearing cells are among those that express RAG genes, Indicating that maturation of $\mathrm{T}$ cells takes place In this organ. In additlon, transfer experimente reveal that lymphold precursors are present in the lung. The stage of differentlation of these precursors will be characterized In future studies. The data presented here Indicate that pulmonary $T$ lymphocytes are derived from both migrants of thymic origin and from precursors which have undergone differentiation and selection in the lung. The population that ls generated in situ and that has not been selected in the thymus may Include cells that are typical for the pulmonary environment.
\end{abstract}

\section{Introduction}

The selection of some $\gamma \delta$ T cell subsets occurs in the lung and is dependent on the genetic background of the mouse strain but not on thymic selection $(1,2)$. This suggests that $\gamma \delta$ T cells in the lung are not necessarily descendants of thymocytes. Notably, in contrast to other peripheral lymphoid organs, there is an unusually high frequency of CD4-CD8- double-negative $\alpha \beta$ $\mathrm{TCR}^{+} \mathrm{T}$ cells among resident pulmonary lymphocytes (3). $\gamma \delta$ and $\alpha \beta$ T cells are also found in the spleen and intestine of athymic nude mice $(4,5)$. Bone marrow reconstitution studies using adult thymectomized mice as recipients have demonstrated the thymic independent origin of intestinal intraepithelial lymphocytes $(5,6)$. It is not clear whether these extrathymically generated $\mathrm{T}$ cells are derived from stem cells which home to the organ, rearrange their receptor genes and proceed to differentiate into T cells, or whether rearrangement and expression of TCR took place prior to migration into the tissue, and only selection and functional maturation occur upon homing. In support of the latter, rearrangements of TCR $\gamma$ genes have been observed in day 11 fetal liver, before the thymus is colonized with $T$ cells $(7)$.
Conceivably, in normal mice, rearrangements of TCR $\gamma$ genes may precede the homing of preT cells to the thymus in some instances and that such cells may already be committed to differentiate along a particular lymphoid lineage. It is also plausible that these pre-committed precursors may be the source of extrathymically generated T cells. In this report, we present data bearing on the origin of resident lymphocytes in the lung, and assess the contribution of the thymic and extrathymic components in the generation and selection of $\mathrm{T}$ cells in this micro environment.

\section{Methods}

Mice

BALB/c mice and BALB/c nu/nu mice were all of Jackson origin and were either purchased from the Jackson Laboratory (Bar Harbor, ME) or from IFFA (L'Arbresle, France). Timed pregnant $B A L B / c$ mice were either purchased from Jackson or from Ciba 
(Basel, Switzerland). The day of birth was considered day 1 of life. CB-17 SCID mice were bred at the Basel Institute for Immunology.

Preparation of resident pulmonary lymphocytes (RPL), thymocytes and splenocytes

To prepare RPL, regardless of the age of the mice, lungs were extensively perfused through the pulmonary artery to remove circulating blood, as previously described (3). The dissected lungs were then cut into $1 \mathrm{~mm}$ strips in PBS without calcium and magnesium and in the presence of $1 \mathrm{mM}$ EDTA at $4^{\circ} \mathrm{C}$. Cells were released from the matrix by teasing the tissue on a steel mesh. The debris was allowed to settle and the cell suspension was washed twice in Iscove's modified Dulbecco's medium in the presence of $5 \%$ FCS. Viable cells were further purified by Ficoll-Hypaque centritugation. Single cell suspensions were also prepared from spleens and thymus by disruption of the organ on a steel mesh. In the case of splenocytes, red cells were removed by hypotonic lysis.

\section{RNA preparations}

Total cellular RNA was extracted from various cellular populations by the APGC procedure (8). For RNA from $\alpha \beta^{+}$or $\gamma^{\delta} \delta^{+}$RPL, cells were magnetically sorted by treatment with biotin-conjugated anti- $\alpha \beta$ TCR mAb H57-597 or anti- $\delta$ antibody GL3, followed by streptavidin-coupled Dynal beads. Trace amounts of contaminating DNA was further removed by treatment with RNAse free DNAse (Boehringer, Mannheim, Germany).

CDNA synthesis and polymerase chain reaction (PCR) reactions

This was performed essentially as published $(2,9,10)$. For PCR analysis of $V_{\gamma}$ gene expression, the sequences of all primers used were as reported, under conditions previously established (9). The RAG-1 and RAG-2 primers for PCR were as described (10). Each PCR cycle consists of the following: $94^{\circ} \mathrm{C} 30 \mathrm{~s}, 52^{\circ} \mathrm{C}$ $30 \mathrm{~s}\left(60^{\circ} \mathrm{C}\right.$ for the RAG 1 and 2 primers), and $72^{\circ} \mathrm{C} 2 \mathrm{~min}$. A 6 min incubation at $72^{\circ} \mathrm{C}$ was added to the last cycle and the number of cycles varied according to the nature of the experiment. In estimating the relative levels of $V_{Y}$ gene expression, to ensure the linearity of the PCR reaction at the time of assay, $5 \mu$ samples were withdrawn at cycles $21,24,27$ and 30 , and the cycles at which the most prominent product became visible was chosen for quantitation.

mAbs

The following mAbs used in this work were purchased from PharMingen (San Diego, CA) anti- $\alpha \beta$ TCR, H57-597; anti- $\delta$ TCR, GL-3; anti- $\mathrm{V}, 4$, UC3-10A6; anti-CD3, 145-2C11; anti-H2K AF6-88.5. M1/70.15 (anti-Mac 1), 187.1 (anti-mouse $x$ ) and 3JP (anti-1-A $A^{b}$ ) were prepared in our laboratory.

\section{Assays for GYS versus GxYS $V_{\gamma} 4$ sequences}

$\mathrm{J}_{\gamma} 1$ primers (10 pmol) were end-labeled with [ $\gamma^{32}$ P]ATP using T4 polynucleotide kinase and purified on a Biorad Bio-spin 6 column. Then, $1 / 10$ th of this was added to a $30 \mu \mathrm{PCR}$ reaction containing as template either $1 \mathrm{ng}$ of boiled plasmid DNA or $10^{4}$ cell equivalent of RPL CDNA, PCR buffer, $100 \mu \mathrm{M} d X T P, 0.5 \mu \mathrm{M}$ $5^{\prime}\left(\mathrm{N}_{\gamma} 4\right)$ and $3^{\prime}\left(\mathrm{J}_{\gamma} 1\right)$ primers, and 0.5 units of Taq polymerase. Amplification was for 25 cycles at $94^{\circ} \mathrm{C} 30 \mathrm{~s}, 50^{\circ} \mathrm{C} 30 \mathrm{~s}, 72^{\circ} \mathrm{C}$ $1 \mathrm{~min}$, followed by an additional $6 \mathrm{~min}$ incubation at $72^{\circ} \mathrm{C}$.
Amplified products were further purified on a Biorad Bio-spin 30 column and an aliquot containing $10^{4}$ c.p.m. from each sample was digested with 5 units of Mboll at $37^{\circ} \mathrm{C}$ for $1 \mathrm{~h}$ in the presence of $0.5 \mu \mathrm{g}$ carrier DNA. Samples were then phenot extracted, ethanol precipitated and solubilized in $5 \mu$ of formamide DNA sequencing gel buffer, heated at $90^{\circ} \mathrm{C}$ for $1 \mathrm{~min}$, and $1 \mu$ was loaded in each lane.

Quantification of RAG-1 concentrations relative to normalized actin concentrations

Total RNA from spleen, thymus and RPL was prepared by the APGC procedure as described above from pools of at least 10 mice. CDNA was synthesized using hexanucleotide primers, starting with RNA extracted from an equivalent of $5 \times 10^{5}$ cells per sample. To estimate the concentration of actin in each preparation, we took $1 \%$ of the CDNA, made a series of 3-fold dilutions, and performed 25 cycles of PCR with $\beta$-actin primers CATCACTATTGGCAACGAGC, ACGCAGCTCAGTAACAGTCC which spanned an intron-exon junction. Then, $1 \mu \mathrm{Ci}$ of [ $\left.{ }^{32} \mathrm{P}\right] \mathrm{dCTP}$ was added to each reaction and the amount of radioactivity incorporated into the $\beta$-actin band was determined after gel fractionation of the PCR products. Adjustments were then made to normalize the quantity of mRNA in the various samples, using actin as an internal standard. Sets of three samples, i.e. spleen, thymus, and RPL from the same mice, were normalized to each other at any one time. The normalization was verified by comparing the amount of radioactivity incorporated into amplified materials obtained for three different concentrations of actin after 20 cycles of PCR amplification. To compare the level of RAG-1 mRNA expression in the different cellular populations using actin as an internal standard, equivalent amounts of actin-normalized CDNA samples were used in PCR amplifications of RAG-1. Typically, a $100 \mu$ reaction was set up including $100 \mu \mathrm{M}$ of each dXTP, $10 \mu \mathrm{Ci}$ [32 P]dCTP, CDNA, PCR buffer, 2 units of Taq polymerase, and $0.5 \mu \mathrm{M}$ of each of the $3^{\prime}$ and $5^{\prime}$ primers. This was split into eight aliquots of $12 \mu \mathrm{l}$ each. $30 \mu$ of oil was layered over each sample, and an aliquot was removed after each increment of three cycles starting from cycle 15 and incubated for another $6 \mathrm{~min}$ at $72^{\circ} \mathrm{C}$ to ensure completion of chain extension. Samples were analyzed on $8 \%$ native polyacryalmide gels and the radioactivity associated with the RAG-1 bands was quantitated on the Phosphor Imager. For each sample, the amount of radioactivity incorporated after different numbers of cycles of amplification was graphed (11).

\section{Assay for lymphoid precursors in the lung}

RPL from $202-3$ week old C57BL6 mice were isolated after extensive perfusion as described. The cells were then incubated with biotin-conjugated anti-CD3, anti- $\alpha \beta$ TCR, anti- $\gamma \delta$ TCR and anti-I-A $A^{b}$ at $4^{\circ} \mathrm{C}$ for $30 \mathrm{~min}$, washed, and incubated with streptavidin-coupled Dynal beads at a bead:cell ratio of 20:1. After a further incubation of $20 \mathrm{~min}$ at $4^{\circ} \mathrm{C}$ with gentle rocking, the antibody bound $T$ cells, $B$ cells, macrophages and dendritic cells were magnetically removed. A second round of depletion was performed and a small fraction of the cells recovered were monitored for purity after further incubation with the antibody cocktail followed by streptavidin - phycoerythrin. Less than 196 of the cells were positive for the combination of markers. Then, $10^{5}$ cells were mixed with $5 \times 10^{5}$ bone marrow cells of SCID origin and injected i.v. into three SCID mice that had been 
(a)

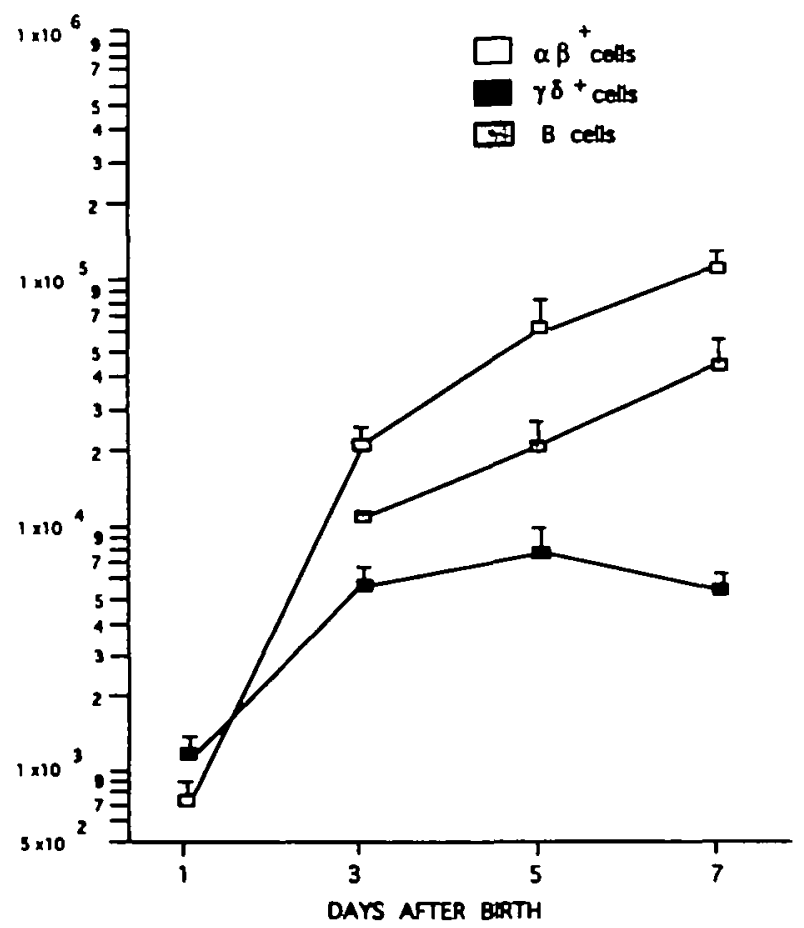

(b)

aphos $T$ cell ratio in aewborn (tay I) BALBk mice

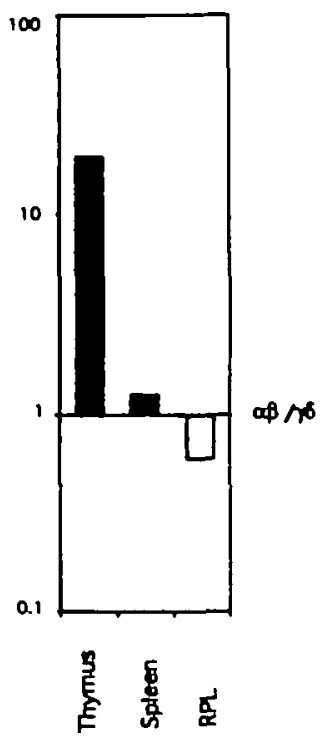

Fig. 1. Lymphocyte colonization of the lung at birth and in the perinatal period. (a) Kinetics of $\alpha \beta$ and $\gamma \delta T$ cell cotonization. (b) Ratio of $\alpha \beta$ versus $\gamma \delta T$ cells in the thymus, spleen and lung at birth. $\alpha \beta$ and $\gamma \delta$ T cells were quantitated by flow cytometric analysis using anti- $\alpha \beta$ TCR (H57-597) and anti-ro TCR (GL-3) antibodies. For each time point, two to four independent measurements were made on pooks of RPL Isolated from five to nine mice. The results are calculated as the number of cells recovered per animal.

previously tested for the absence of serum lg. The SCID mice were 3 months old at the time of transter and were irradiated (350 rad) before injection. Control mice received only SCID bone marrow. Four months later, the mice were killed, and cells were isolated from their spleens, thymus and lungs for analysis.

\section{Results}

$T$ cell colonization of the lung during the first woek of life

To analyze the kinetics of T cell colonization of the lung during the perinatal period, we used a standard method for the isolation of RPL. On average, immediately after birth, we recovered a total of $>10^{3} \gamma \delta$ T cells from the lungs of each mouse, but only $7 \times 10^{2} \alpha \beta$ T cells (Fig. 1a). At the same time, in the thymus and spleen, the number of $\alpha \beta T$ cells exceeded that of $\gamma \delta$ T cells (Fig. 1b). These observations suggest that either the $\gamma \delta$ T cells present in the lung at birth are generated in situ and do not come from the thymus or that there is a preferential homing of $\gamma \delta T$ cells to the lung before birth. However, after birth, between day 1 and 3 , the number of $\alpha \beta$ T cells increases by almost 10-fold in the lung, while $\gamma \delta$ T cells increase less dramatially in number. The $\gamma \delta: \alpha \beta$ ratio is rapidly reversed during the first week of life, such that by day $7, \gamma \delta$ T cells represent a minor fraction of RPL (Fig. 1). Thus, in the first week after birth, there is a rapid colonization of the lung by $\alpha \beta T$ cells, while the number of $\gamma \delta$ $T$ cells increases slowly.
Age related changes in the pattern of $V_{\gamma}$ gene expression The high frequency of $\gamma \delta$ RPL at birth compared with $\alpha \beta$ T cells prompted us to investigate the $V_{r}$ gene usage in this population. This was performed by a modified PCR-based assay that has previously been used to define the pattern of $V_{\gamma}$ and $V_{s}$ genes expressed in RPL isolated from adult BALB/C mice $(1,2,9)$. Surprisingly, the predominant $V_{\gamma}$ gene that was expressed in the lung at birth was $V_{Y} 6$ (Fig. 2a). We have previously shown that the major $V_{\gamma}$ mRNA species present in adult BALB/c RPL was $V_{\gamma} 4$ (2), but that in mice immunized with aerosols of purified protein derivative, both $V_{\gamma} 4$ and $V_{\gamma} 6$ cells are present (9). Moreover, a large fraction of the resident lymphocytes isolated from the adult lung reacts to the anti- $V_{4} 4$ antibody UC3-10A6 (12) that has become available (unpublished observation). The conspicuous absence of the $V_{\gamma} 4$ population in the neonatal lungs prompted us to analyze the kinetics of expression of the various $V_{\gamma}$ genes in RPL isolated from mice of different ages.

As shown in Fig. 2 , in very young mice, the pattern of $V_{\gamma}$ gene expression in RPL is significantly different from that of the adult. It is also different from that of the thymus and spleen in mice of the same age. Previous studies have shown that right before birth and in adult mice, $V_{\gamma} 4$ is the main $V_{\gamma}$ gene that is expressed in the thymus, lymph node and spleen $(13,14)$. However, in the lung, from birth until the young adult period, $v_{y} 6$ constitutes the major fraction of the $\gamma \delta T$ cells present, although $V_{Y} 4$ bearing cells eventually predominate in older animals (Fig. 2a). 

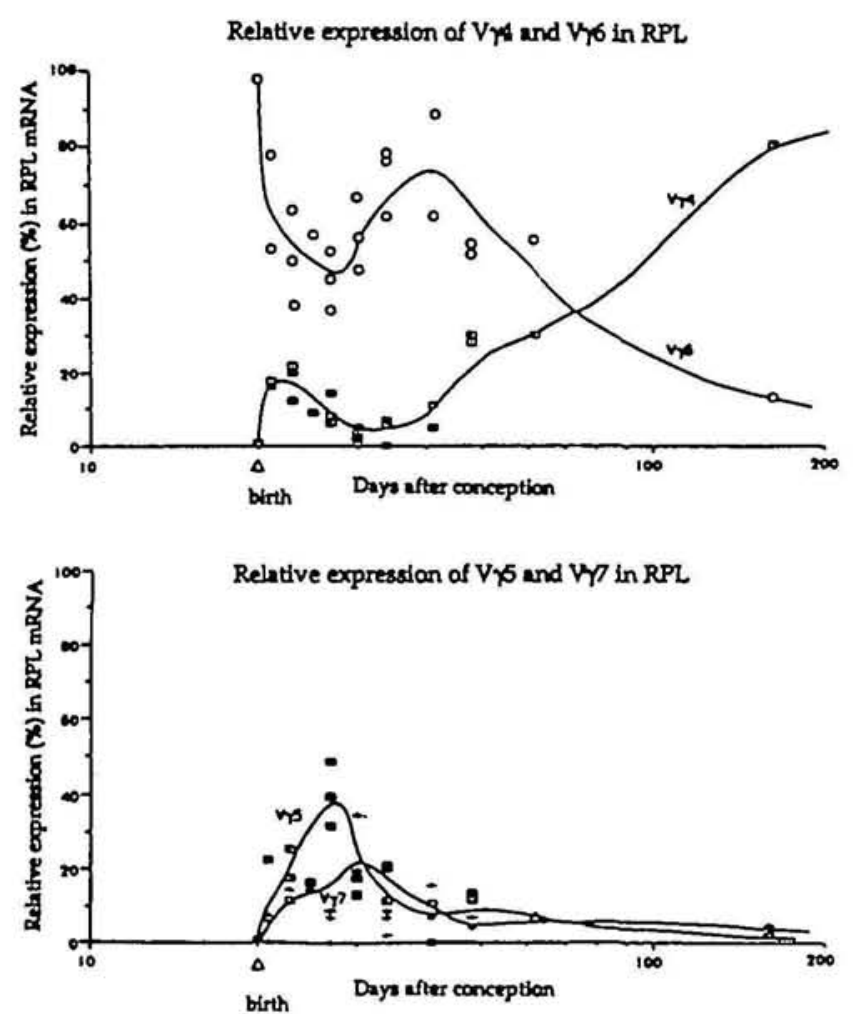

Flg. 2. $V_{r}$ gene expression in RPL changes with time. (a) Relative expression of $V_{\gamma} 4$ and $V_{\gamma} 6$. (b) Retative expression of $V_{\gamma} 5$ and $V_{\gamma} 7$. Each data point was obtained from RNA trom a pool of at least five mice. Different data points for mice of the same age were derived from different batches of mice. For each sample of RPL, equal amounts of the same preparation of $\gamma$ CDNA were used as templates for the amplification of the four different $V_{\gamma}$ genes under conditions of linear amplification (see Methods). The PCR products of V 4, 5, 6 and 7 were fractionated on a $1.4 \%$ agarose ged, transferred to Genescreen membrane (Dupont), and hybridized to a ${ }^{32}$ p-labeled $J_{\gamma} 1$ probe. The amount of radioactivity

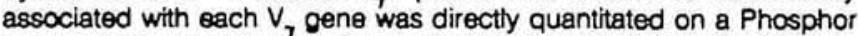
Imager (Molecular Dynamics) or an AMBIS Bioanalysis Imaging System. The relative level of expression of each $V_{\gamma}$ gene was estimated as a percentage of all $V_{\gamma}$ genes assayed for that time point. The $y$-axis represents the percentage gene expression on a linear scale. The $x$-axis represents the time elapsed after conception of the fetus on a $\log _{10}$ scale. The curves representing the kinetics of relative expression for each $V$ gene were obtained by interpolation. The four graphs were plotted on two separate figures to allow better discrimination of the data points.

There is a first peak of $V_{\gamma} 4$ expression right after birth. This drops to a very low level in $2-3$ week old mice, but increases steadily thereafter such that the majority of $\gamma \delta T$ cells in older BALB/c mice express $V_{\gamma} 4$ by 5 months of age. The data on $V_{\gamma} 4$ have been verified by surface staining using a $V_{\gamma} 4$-specific antibody. There is a strong correlation between the RNA and the immunoflorescence data (data not shown). $V_{\gamma} 5$ and $V_{\gamma} 7$ expression each emerges as a wave that peaks respectively on day 7 and 10 after birth, but constitutes a very low proportion of $\gamma \delta$ RPL later in life. In this study, the relative levels of $\mathrm{V}_{\gamma} 1 \mathrm{C}_{\gamma} 4$ mRNA are barely detectable throughout. The data plotted indicate that changes in the ratios between these populations occur as a function of time and that, in general, waves of $\gamma^{\delta} \mathrm{T}$ cells expressing distinct $V_{\gamma}$ genes appear in the lung at various time points after birth.

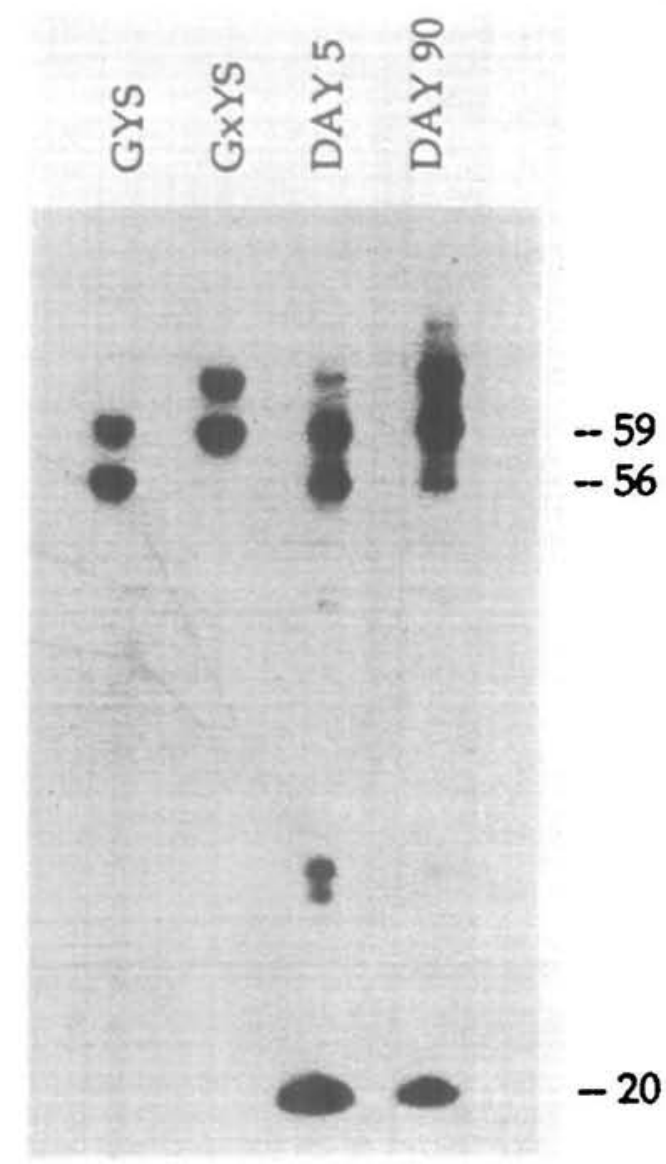

Fig. 3. The first and second waves of $V_{\gamma} 4$ cells which colonize the lungs differ in their predominant TCR sequences: GYS versus GXYS. Autoradiogram of an $8 \%$ denaturing polyacrylamide gel showing the patterns of Mboll cleaved PCR amplified products from (a) a plasmid carrying a $V_{\gamma} 4$ cDNA clone with a GYS junction, (b) a plasmid carrying a $V_{\gamma} 4$ cDNA clone with a GXYS junction, (c) total CDNA prepared from day' 5 BALB/c RPL and (d) total CDNA prepared from 3 month old BALB/c RPL. An anti-sense $32 \mathrm{P}$-nd-labeled $J_{\gamma} 1$ primer $\left(5^{\prime}\right.$-CCTCTGCAAATACCTTGTG) was used in the amplification together with unlabeled $V_{\gamma} 4$ primer. Mboll does not cut at the recognition site itself but 8 bases downstream of it. Due to two closely spaced Mboll recognition sites, the expected Mboll fragments for GYS-type junctions are 56 and 59 bases respectively, and those for GXYS-type junctions are 59 and 62 bases. The 20 nucleotide band is due to contaminating $\mathrm{J}_{\gamma} 1$ primers.

Why does the first peak of $\mathrm{V}_{\gamma} 4$ decline while the second wave of $V_{\gamma} 4$ continues to expand? One possibility is that the first set of cells is qualitatively different from the second, late-appearing population. To test this, we took advantage of the fact that the predominant $V_{\gamma} 4 \mathrm{~J}_{\gamma} 1$ rearrangement is represented in older BALB/c RPL by GXYS sequences, while GYS is predominant among 'fetal' $V_{\gamma} 4$ rearrangements $(2,15)$. To determine the type of TCR sequence that prevails among the $\mathrm{V}_{\gamma} 4$ rearrangements in the two populations, we measured the sizes of the $\mathrm{V}_{\gamma} 4 \mathrm{~J}_{\gamma} 1$ junctions. Using a ${ }^{32} \mathrm{p}$-end-labeled $\mathrm{J}_{\gamma} 1$ primer, we amplified the $\mathrm{V}_{\gamma} 4$ cDNA from RNA extracted from day 5 (representing peak 1) and 90 (representing peak 2) RPL, digested the PCR product with the diagnostic restriction enzyme Mboll, and analyzed the labeled fragments. It is evident from Fig. 3 that the first peak consisted mainly of GYS-type junctions which are three nucleotides shorter than that of the GXYS type that predominates in the 


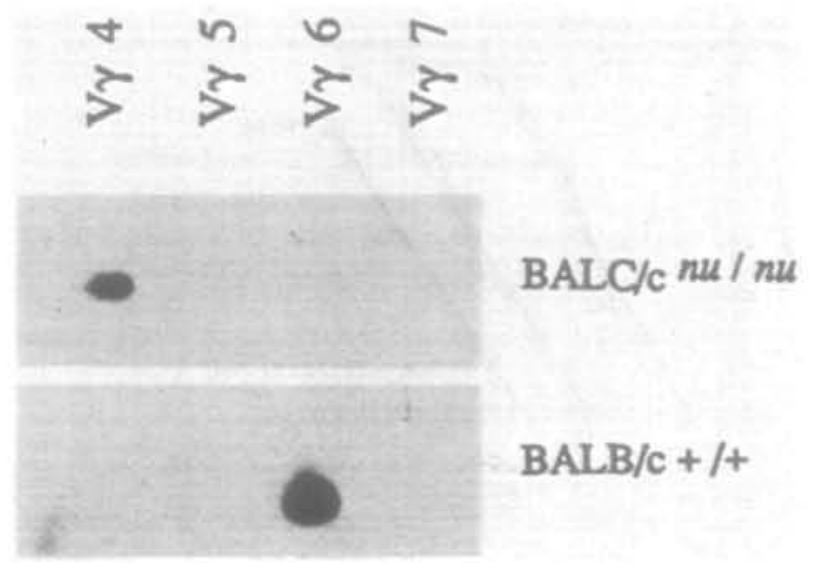

Fig. 4. Comparison of $V_{\gamma}$ gene expression in newborn BALB/c and BALB/c nulnu RPL. CDNẢs corresponding to $10^{5}$ non-adherent cells isolated from newborn BALB/c or newborn BALB/c nude mice (verified by their lack of the thymus) were divided into four aliquots and used as templates for PCR amplification of $V, 4,5,6$ and 7 in conjunction with the $\mathrm{C}_{\gamma} 1$ primer. Thirty cycles of PCR were performed, and the PCR products were fractionated by gel electrophoresis and hybridized to a ${ }^{32} \mathrm{P} 5^{\prime}$-end-labeled $J_{\gamma} 1$ primer.

second peak. Thus, the variation in $V_{\gamma} 4$ expression in early life is due to the appearance of a peak of 'fetal type' $V_{\gamma} 4$ bearing cells, several days after the shift to the 'adult' type of rearrangements had already occurred in the thymus. These data could be explained, if one assumes that some of these T cells are not thymus derived or that the pulmonary environment early in life does not allow the selective expansion of adult $V_{\gamma} 4 T$ cells, but transiently favors the expansion of cells bearing the 'fetal' GYS receptor. Alternatively, a combination of both factors may be responsible for our observation.

\section{Thymic origin of $V_{\gamma} 6$ in RPL in newborn mice}

Data from previous work have shown that $V_{\gamma} 4$ RPL can be generated in the absence of the thymus (2). However, it has also been shown that the generation of $\mathrm{dEC}$ of the skin which bore the $V_{\gamma} 5$ canonical receptor is strictly thymus dependent (16). It was therefore of interest to determine whether the $V_{\gamma} 6 T$ cells found in the lung at birth require the presence of the thymus for their appearance. Therefore, we compared the nature of the $\gamma \delta$ TCR genes expressed in RPL isolated from newborn BALB/C mice with those of newborn BALB/c nulnu mice. As shown in Fig. 4 , in the absence of the thymus, the $V_{\gamma} 6$ population is not detectable in the lung at birth and that the few $\gamma \delta$ T cells present at this time are mainly of the $\mathrm{V}_{\gamma} 4$ subset. This establishes that at least one major subset of $\gamma \delta \mathrm{T}$ cells in the lung of normal mice is of thymic origin and that the lung is also a preferred site where $\gamma \delta T$ cells home to during ontogeny.

\section{High levels of RAG-1 and RAG-2 expression in the lung}

The marked asynchrony in the TCR phenotype between cells that are produced in the thymus and those that home to the lung may be explained as follows. The lung is populated in early life with $T$ cell precursors or immature $T$ cells of thymic or non-thymic origin, all of which are programmed to generate subsets of lymphocytes in a preset sequence upon receiving the appropriate signals. These hypothetical precursors, at the time of their

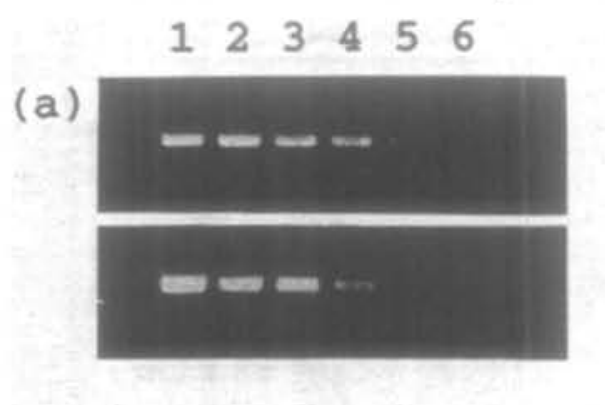

RAG-1

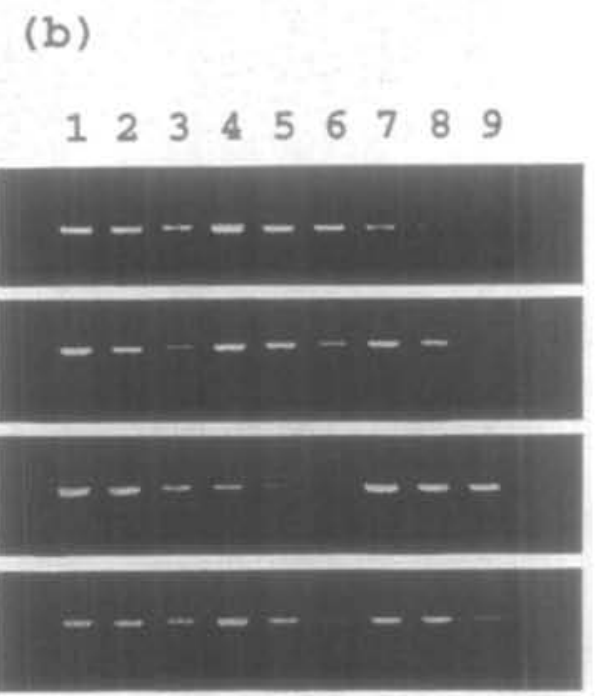

NEWBORN

DAY 3

DAY 5

DAY 11

Fig. 5. RAG-1 and RAG-2 are present in RPL of young BALB/c mice. (a) Detection of RAG-1 and RAG-2 expression. In lanes 1-6, RNAs from $6.25 \times 10^{5}, 1.25 \times 10^{5}, 2.5 \times 10^{4}, 5 \times 10^{3}, 10^{3}$ and $2 \times 10^{2}$ thymocytes from 4 week old mice were analyzed using 30 cycles of PCR. One third of the amplified products were shown in the ethidium bromide stained gels. (b) RAG-2 expression in thymocytes, RPL and splenocytes from mice of various ages. Lanes $1-3$ represents the RAG-2 PCR products from $10^{4}, 3 \times 10^{3}$ and $10^{3}$ thymocytes; lanes $4-6$ and $7-9$ represents the RAG-2 PCR products from $10^{6}, 3 \times 10^{5}$, and $10^{5}$ splenocytes and $\mathrm{RPL}$ respectively.

migration to the lung, might not have completed gene rearrange. ment, or have not yet undergone selection, and might express RAG-1 and RAG-2 genes while in the lung. To investigate this possibility, we examined total RNA from newborn, day 3, day 5 and day 11 thymocytes, spleen and RPL for RAG-1 and RAG-2 activities. To begin with, we performed a series of 5 -fold dilutions of newborn thymocyte $\mathrm{CDNA}$ beginning with $10^{5}$ cell equivalent, and determined that we could easily detect both RAG-1 and RAG-2 activities among $10^{3}$ thymocytes on ethidium bromide stained gels after 30 cycles of PCR amplification (Fig. 5). In this population, the level of RAG-1 RNA was $\sim 5$-fold higher than that of RAG-2. We then proceeded to determine whether the RAG-1 and RAG-2 genes were expressed in RPL, and discovered that, indeed, such mRNA species were found in RPL preparations from newborn, day 3 , day 5 and day 11 mice, but at lower abundance than in the thymocyte population (Fig. 5 b).

To assess the significance of this observation, we used a quantitative PCR method to compare the amount of RAG-1 RNA expressed in thymocytes, splenocytes and RPL at various ages 

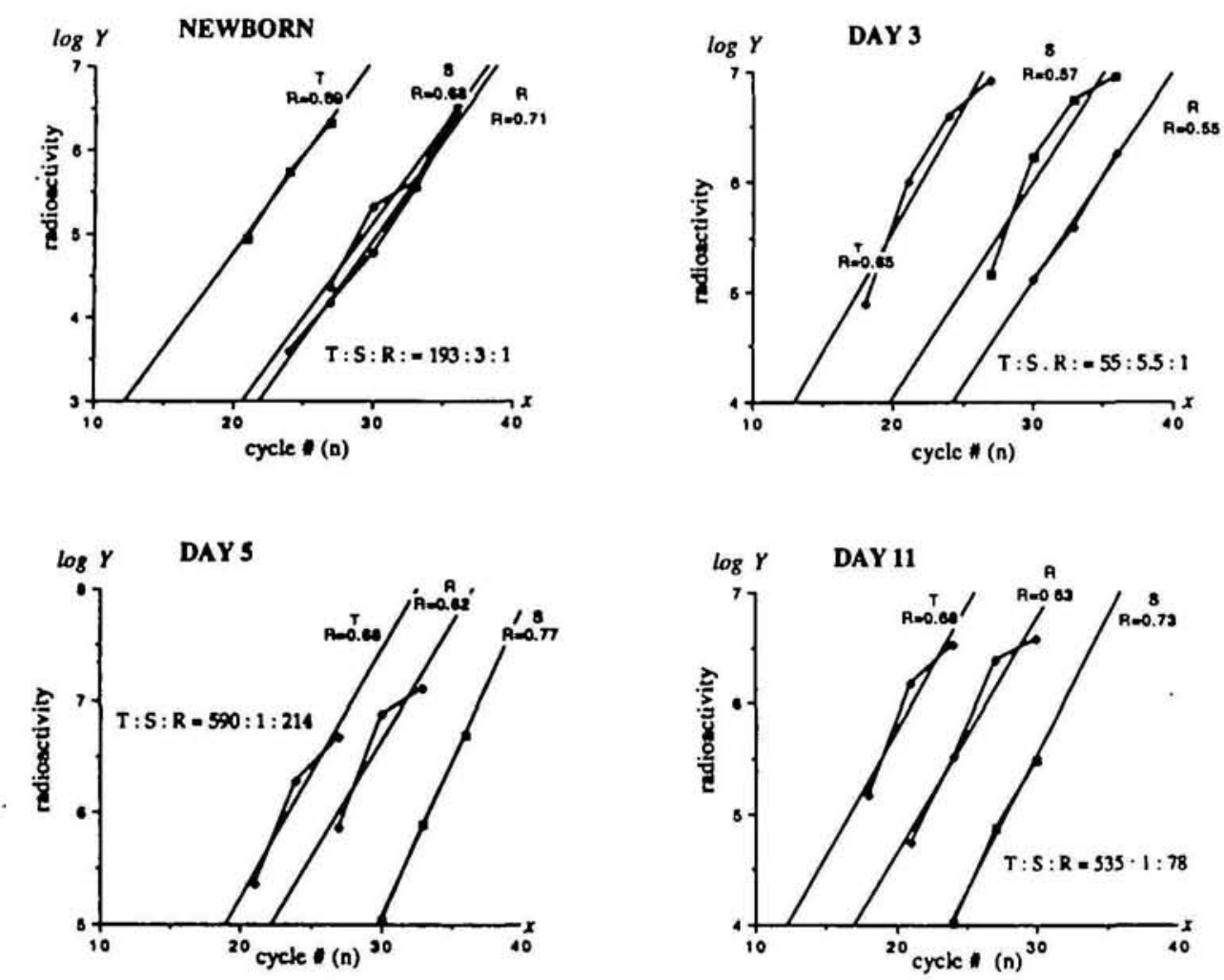

Fig. 6. Relative levels of RAG-1 expression in thymocyte, splenocytes and RPL populations at various time after birth. Splenocytes (S), thymocytes (T) and RPL (R) were isolated from mice of different ages and the quantitative PCR performed as described in Methods. The extent of amplification $\left(\eta\right.$ is given by the formula $Y=A(1+R)^{n}$, where $A$ is the initial amount of RAG.1 transcript, $R$ is the efficiency of the amplification procedure and $n$ is the number of PCR cycles (11). $Y$ can be estimated by the amount of radioactivity incorporated into the RAG-1 band. The plot $\log Y=\log A+n$ $\log (1+)$ with $\log Y$ as a function of $n$ yields a slope value $=\log (1+R)$ from which $R$ is derived. Thus the initial value $A$ can be calculated and the relative initial quantity of RAG.1 mRNA estimated. The ratio of the $A$ value is shown for each set of comparison.

in the perinatal period. The results are plotted in Fig. 6 . Since the absolute amount of RAG-1 mRNA per cell cannot be measured, the relative level of RAG-1 expression is calculated using $\beta$-actin as an internal standard. As expected, at all ages tested, thymocytes show the highest level of RAG-1 activity. Surprisingly, atthough RAG-1 was expressed in RPL at levels lower than in splenocytes at days 1 and 3 after birth, the level of RAG-1 mRNA in RPL was significantly higher than in splenocytes at day 5 and 11: by 200 - and 80-fold respectively (Fig. 6). Furthermore, RAG-1 and RAG-2 transcripts can both be found in the TCR $\alpha \beta^{+}$as well as the TCR $\gamma^{+}{ }^{+}$populations isolated from the lung (Fig. 7). Since mature T cells that have undergone selection are expected to shut off RAG gene expression $(17,18)$, these data support the idea that the T cell repertoire in RPL is shaped at a significant extent by local diversification and selection.

Precursor potential of antigen receptor negative cell in RPL

The T cells in the lung which express RAG could be either thymic migrants that have not yet undergone selection or they could be derived from $T$ cell precursors present in the lung. To further investigate these possibilities, we injected 3 month old SCID mice with cells isolated from C57BL/6 RPL that were depleted of T and $B$ lymphocytes, and asked whether there were any changes in the lymphoid compartment of the recipients 4 months later. Each irradiated CB-17 SCID mice $\left(\mathrm{H}-2^{\circ}\right)$ received $10^{5}$ antigen receptor negative cells from $2-3$ weeks old C57BL/6 $\left(\mathrm{H}-2^{\circ}\right)$

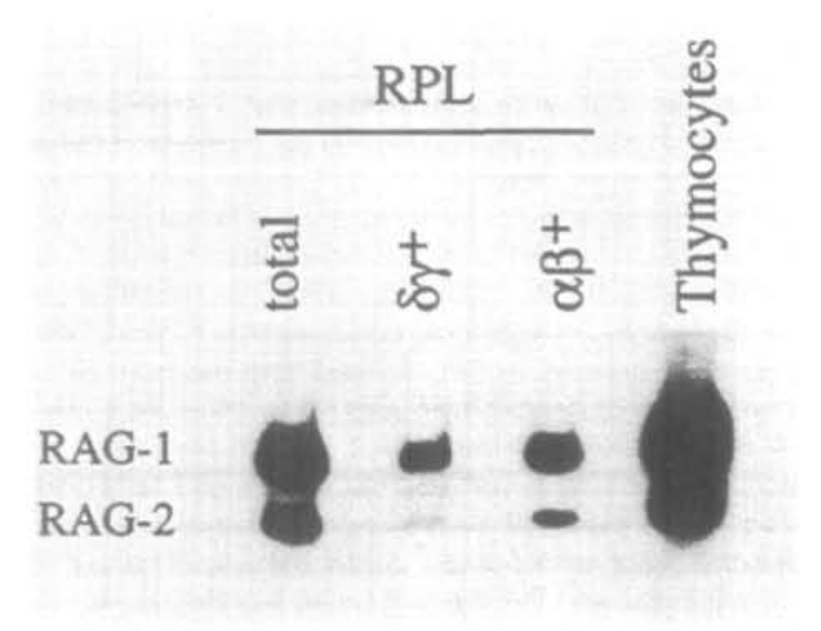

Fig. 7. RAG-1 and RAG-2 are expressed in $\gamma \delta$ and $\alpha \beta$ surlace TCR positive cells. RPL $\left(2 \times 10^{6}\right)$ from 3 week old BALB/c mice were pre-incubated with the anti-Fc block antibody from PharMingen for 15 $\mathrm{min}$ at $4^{\circ} \mathrm{C}$, divided into three fractions and either treated with biotin. conjugated anti-y $\delta$ TCR antibody (GL-3), anti- $\alpha \beta$ TCR antibody $(\mathrm{H} 57.597)$ or medium onty for $30 \mathrm{~min}$. TCR cells were isolated using streptavidincoated Dynal beads and RNA was prepared from the bead bound cells. RAG-1 and RAG-2 expression were analyzed in the same reaction using $32 \mathrm{p} 5$ 'end-tabeled primers, and the reaction products were fractionated on a $6 \%$ polyacrylamide gel A PCR reaction using a thymocyte derived template was performed in parallel. 
Table 1. Phenotypic analysis of splenocytes and RPL trom SCID mice which received precursors from the lung

\begin{tabular}{|c|c|c|c|c|c|c|c|c|c|}
\hline \multirow[t]{3}{*}{ Mouse } & \multicolumn{4}{|l|}{ RPL } & \multicolumn{5}{|c|}{ Spleen } \\
\hline & \multirow[t]{2}{*}{$\mathrm{H} \cdot 2^{\mathrm{b}}$} & \multicolumn{3}{|l|}{$\mathrm{H}-2^{b}$} & \multirow[t]{2}{*}{$H \cdot 2^{b}$} & \multicolumn{4}{|l|}{$\mathrm{H}-2^{\mathrm{b}}$} \\
\hline & & $\mathrm{CD} 3$ & $\alpha \beta$ & $\gamma \delta$ & & $\mathrm{CD} 3$ & $\alpha \beta$ & $\gamma \delta$ & $\lg x$ \\
\hline $\begin{array}{l}\text { Control } \\
\text { SCID } 1 \\
\text { SCID } 2 \\
\text { SCID } 3 \\
\text { SCID } 4 \\
\text { SCID } 5 \\
\text { SCID } 6\end{array}$ & $\begin{array}{c}1.2 \\
79 \\
2.0 \\
53 \\
40 \\
34 \\
71\end{array}$ & $\begin{array}{c}1.0 \\
74 \\
3.2 \\
42 \\
7.3 \\
6.4 \\
59\end{array}$ & $\begin{array}{c}0.7 \\
67 \\
1.3 \\
33 \\
3.2 \\
1.7 \\
49\end{array}$ & $\begin{array}{l}0.5 \\
2.3 \\
0.8 \\
8.8 \\
4.8 \\
8.2 \\
5.0\end{array}$ & $\begin{array}{l}0.8 \\
76 \\
37 \\
25 \\
57 \\
54 \\
40\end{array}$ & $\begin{array}{c}1.2 \\
64 \\
4.2 \\
24 \\
4.7 \\
5.0 \\
19\end{array}$ & $\begin{array}{c}0.6 \\
60 \\
1.1 \\
20 \\
1.6 \\
1.7 \\
13\end{array}$ & $\begin{array}{l}0.5 \\
4.6 \\
4.1 \\
4.0 \\
3.8 \\
4.5 \\
5.4\end{array}$ & $\begin{array}{c}1.2 \\
6.5 \\
31 \\
2.3 \\
50 \\
39 \\
26\end{array}$ \\
\hline
\end{tabular}

SCID nos $1-3$ and $4-6$ are trom two sets of independent experiments. The control is representative of the six SCID mice which received only SCID bone marrow cells after irradiation. The data were collected on a FACScan. The anti $\mathrm{H}-2^{b}$ antibody was conjugated to biotin and revealed with streptavidin-phycoerythrin. The other reagents were FITC conjugates. Wherever applicable, the anti-l $x$ staining was always followed by a $30 \mathrm{~min}$ incubation with $30 \%$ mouse serum before the addition of another antibody. The data presented represent percentage of total celts analyzed.

donors, together with $5 \times 10^{5}$ SCID bone marrow cells as a source of hematopoietic precursors. Control mice were given only SCID bone marrow cells. Two independent experiments were performed, each consisting of groups of three recipients. As shown in Table 1, cells of $\mathrm{H}-2^{\mathrm{b}}$ donor origin were present in each of the six recipients which received cells purified from RPL, while no $\mathrm{H}-2^{\circ}$ cells were found in mice which received only SCID bone marrow. The presence of both $T$ and $B$ cells of donor origin indicates that more than one lineage of lymphocytes can be generated from the cells transferred.

The thymus, spleen and RPL from each reconstituted SCID mouse were individually analyzed. We recovered $0.5-1 \times 10^{6}$ cells from the thymus, but there were no lymphoid cells present according to forward and side scatter flow cytometry analysis, and no $\mathrm{H}-2^{\mathrm{b}}$ positive cells were detected in the thymic preparations. From the spleens, we obtained $0.6-2 \times 10^{7}$ cells and $\mathrm{H}-2^{\mathrm{b}}$ positive lymphocytes were present in all cases. From the lungs, $0.6-1.5 \times 10^{5} \mathrm{RPI}$ were obtained. With one exception (no. 2), all mice displayed a significant fraction of $\mathrm{H} \cdot 2^{\mathrm{b}}$ positive RPL. In spite of the undetectable level of $\mathrm{H} \cdot 2^{b}$ positive cells in the lung of SCID no. 2,37\% of the splenocytes in this mouse were of donor origin. A summary of the phenotypic analysis is presented in Table 1. In the recipient SCID no. 1, practically all the $\mathrm{H}-2^{b}$ positive cells were $T$ cells, both in the spleen and in the lung. On the other hand, in SCID no. 2, there were no T cells in either the spleen or the lung, but donor derived B cells were present in the spleen. The other four mice had varying numbers of $T$ and $B$ cells in their spleens and lungs. Moreover, since the presence of a large number of allogeneic cells in the recipient SCID mice do not appear to induce clinically detectable graft versus host disease, one can further argue that these cell are descendants of immature precursors. Considering the low number of cells transferred and the fact that they have been depleted of TCR and cell surface lg bearing cells, these data show that a very good level of lymphoid reconstitution was achieved from the precursor enriched cells isolated from the lung epithelia. It is evident from Table 1 that not all mice have the same number of mature, antigen receptor bearing lymphocytes. Most likely, the number of cells transferred is close to the lower limit at which single precursors can be detected by this assay.

Among the $\mathrm{CD}^{+}$cells, it should be noted that both $\gamma \delta$ and $\alpha \beta T$ cells were present; however, their proportion varies from mouse to mouse. The relative levels of $\gamma \delta$ and $\alpha \beta$ T cells among RPL is represented in Table 1. It appears that $\gamma \delta \mathrm{T}$ cell representation is higher when there are fewer total $\mathrm{CD}^{+}$cells. It also appears that the development of $\gamma \delta$ and $\alpha \beta$ T cells occurred independently. Taken together, these data support the idea that at least part of the $T$ cell repertoire in the lungs of normal mice is generated and selected locally.

\section{Diecusstons}

One of the most intriguing observations in the field of $\gamma \delta \mathrm{T}$ cell biology is the presence of distinct $T$ cell subsets marked by specific TCR rearrangements in different epithelia. While the partitioning of $\gamma \delta T$ cells appears to be a function of the fine structures of their TCR, the nature of the elements which maintain this specific partition is not evident. In the first series of experiments, we attempt to identity a dynamic pattern in the homing of resident $\gamma \delta$ T lymphocytes to the lung epithelia with the hope that it would indicate whether the localization of certain $\gamma \delta \mathrm{T}$ cells in the lung is due to preferential homing or whether it is the product of site specific proliferation.

At birth, $V_{\gamma} 6$ expression predominates in RPL (Fig. 2), but between day 1 and 5 of extra-uterine life, the relative representation of $V_{\gamma} 4, V_{\gamma} 5$ and $V_{\gamma} 7$ transcripts increases, such that, proportionately, $V, 6$ drops to a minimum around day 7 . Since in this period there is only a small increase in the number of $\gamma \delta$ T cells in the lung (Fig. 1), these changes could be due to a local selective expansion of the resident population rather than to new arrivals from the thymus. Surprisingly, further measurements revealed patterns of $V_{\gamma}$ gene expression which were not predicted by this hypothesis or by other views on the preferen. tial $\gamma^{\delta} T$ cell homing in various epithelia. Specifically, $V_{\gamma} 4$ expression describes a first peak soon after birth, drops again in the young adult animal but becomes predominant again in older mice (Fig. 2). $V_{\gamma} 6$ expression displays an inverse correlation with $V_{\gamma}$. This is particularly evident in older mice, where $V_{\gamma} 6$ is progressively replaced by $V_{\gamma} 4$. However, there is a major difference between the $V_{\gamma} 4$ and $V_{\gamma} 6 T$ cells: while both the earty and the late $V, 6$ peaks consist of fetal canonical $\gamma \delta$ TCR, the two peaks of $V_{\gamma} 4$ differ in their TCA composition. The early peak contains mainly fetal-type $V_{\gamma} 4 J_{\gamma} 1$ rearrangements of GYS junctions (2), while the later peak consists mainly of adutt-type GxYS rearrangements (Fig. 3). The unexpected peak of fetal $V_{y} 4$ rearrangements which appears among RPL after bith could be due to 'left-over' fetal thymocytes that were produced between $E 15$ and $E 18$ but only expressed lung-specific homing receptors 5- 10 days after they were generated. Alternatively, one could consider the possibility that a slightly delayed wave of TCR rearrangements might have taken place among immature lymphocytes in the lung epithelia. Thus, the first wave of $V_{\gamma} 4$ expression in the lung could be explained by the extrathymic production of a pool of 'fetal' $V_{\gamma} 4$ bearing cells during extrauterine life, at a moment when the shift to 'adult'-type TCR rearrangements has already occurred in the thymus.

The next set of experiments was designed to test the hypothesis 
that the lung is populated in early life with $T$ cell precursors of thymic or non-thymic origin, programmed to generate $T$ cells in a preset temporal sequence. These precursors might not have completed TCR gene rearrangements at the time of migration to the lung and might express the recombination activating genes, RAG-1 and RAG-2, while in the lung. Indeed, these mRNA were found in RPL preparations at surprisingly high levels early in life. To assess the significance of this observation, we compared the quantity of RAG-1 mRNA expressed in thymocytes and splenocytes with RPL of the same age at various times in the perinatal period. In essence, RAG-1 levels in RPL were lower than in splenocytes at days 1 and 3 after birth, but increased dramatically to levels of 200- and 80-fold higher than in splenocytes at day 5 and 11 respectively (Fig. 6). Such data support the idea that the structure of the T cell repertoire in RPL is shaped in situ rather than by differential homing of individual clonotypes preselected in the thymus. Thus, the existence of the short-lived waves of perinatal if RPL and the data on RAG expression in the lung during this period suggest that colonization of the lung with pre-T cells could occur.

Surprisingly, RAG-1 and RAG-2 transcripts were found in both $\gamma^{\delta+}$ and $\alpha \beta^{+}$RPL (Fig. 7), suggesting that colonization of the lung with $T$ cell precursors or with $T$ cells still capable of performing TCR rearrangements in situ occurred for both lineages. Two explanations of this observation should be considered: (i) that T cell precursors in which rearrangements are still spontaneously taking place are preferentially attracted to the lung epithelium or (ii) that $T$ cells which already exhibit a functional TCR home to this organ, but that upon encounter with the local antigenic environment, their RAG-1 and RAG-2 activity can be modulated. In this second case, their selection could occur by deviating, subsequent to secondary rearrangements, their original antigen specificity. In either case, one wonders whether such cells have the potential to diversity and proliferate efficiently, so that they could serve as lymphocyte precursors in in vivo reconstitution experiments.

The transfer of allogeneic TCR and Ig receptor negative 'RPL' into irradiated SCID mice was informative for assessing the precursor potential of these cells. It appears that, over a long period of time (4 months), they can generate not only a $T$ cell population which contains both the $\alpha \beta^{+}$and the $\gamma \delta^{+}$subsets, but also $B$ cells. The lack of thymic reconstitution in these SCID mice contrasts with the substantial colonization of lymphoid organs and of the lung epithelium with mature lymphocytes. This could be interpreted as an indication of the extrathymic differentiation potential of the $T$ cell precursors isolated from the lung epithelia. The low number of cells $\left(10^{5}\right)$ transferred and the generation of significant numbers of lymphocytes of allogeneic origin indicate that these lymphocytes developed from immature precursors that have a high differentiation and proliferation potential. This pattern of 'active reconstitution' of SCID mice with $T$ cells is in contrast with the relatively low level of in vivo expansion of peripheral adult T cells transferred into SCID mice documented by Sprent et al. (19). In essence, the extrathymic differentiation of some lymphoid precursors in the lung is a reasonable explanation for our data on the early waves of $\gamma \delta T$ cell colonization of the pulmonary epithelia, as well as the high levels of RAG-1 and RAG-2 expression that we observed. In this regard, one should note that the ability of the murine intestine to generate and select its own $T$ cell repertoire is now well documented $(20,21)$.

A recent quantitative investigation of the ordered rearrangements at the $\mathrm{C}_{\gamma} 1$ locus indicates that for individual $V_{\gamma}$ genes there is a strong correlation between transcription and rearrangement frequency (22). Moreover, there are strong indications that the rearrangement machinery in fetal thymocytes favors the production of canonical $V_{\gamma} 5 J_{\gamma} 1$ dendritic-epidermal cell junctions (23). In the perspective of previous work (16) which demonstrated that the high frequency of productive $V_{\gamma} 5$ fetal canonical rearrangement requires both fetal $T$ cell precursors and the fetal thymic environment, these more recent data suggest that $T$ cell precursors are not intrinsically prone to rearrange a particular type of $V_{\gamma}$ gene, but that this event is selectively induced by the thymic stroma. Our data favor the notion that some T cell rearrangements can occur extrathymically, but that, at the same time, they are coordinated with thymic development. This could be achieved if a specific thymic soluble factor and not a thymic stromal cell ligand is responsible for inducing the ordered and coordinated transcription and rearrangement of various $V_{\gamma}$ genes. On the other hand, recent reports showing that the engraftment of fetal skin and intestine promote $T$ cell development $(24,25)$ further demonstrate the thymus-like activity of these epithelial tissues. Conceivably, the lung epithelium may have similar thymopoietic potential.

The role of the thymus in generating part of the pulmonary repertoire is not to be denied. Figure 1 clearly illustrates that the lung is a preferred site for the homing of $\gamma \delta$ T cells during the perinatal period. However, this first set of $V_{\gamma} 6$-specific RPL is undetectable at birth in the absence of the thymus, as in nude mice, where the $\mathrm{V}_{\gamma} 4$ subset is present (Fig. 4). It is also evident that the total number of $T$ cells is much lower in the lungs of adult nu/nu mice, as is the case for the intestinal epithelial hymphocytes (5), when compared with euthymic mice. Thus, it is possible that in euthymic mice certain thymic factors may enhance extrathymic $T$ cell differentiation.

From the data presented and discussed here, it appears that the resident pulmonary $T$ cell population consists of cells which are generated and selected locally, as well as of cells which are generated in the thymus but may be further selected and expanded in the pulmonary environment. Our finding that RAG-1 and RAG-2 are expressed in cells which already have TCRs on their surface is in line with recent reports showing that a significant fraction of peripheral $\gamma \delta$ and $\alpha \beta$ T cells in human blood express two different TCRs $(26,27)$. It is possible that some thymic derived $T$ cells may undergo further TCR mediated selection in the pulmonary environment as part of their adjustments to the requirements of this micro environment. On the other hand, resident pulmonary $T$ cells that are generated extrathymically, and hence have not undergone thymic selection, may include cells carrying specificities that are absent among the thymus selected set but which are unique to the pulmonary environment. In this way, thymic and extrathymically derived RPL may complement each other in the defense of the host.

\section{Acknowledgements}

We are grateful to A. Lanzavecchia, K. Karjalainen and F. Ronchese for critical reading of the manuscript, and M. MOCerty and A. Sir for technical 
assistance. This work was supported in part by National Institutes of Health grants to A. Augustin. The Basel Institute for Immunology was founded and is supported by F. Hoffmann-La Roche Lid, Basel.

\section{Abbreviations}

dEC

PCR

dendritic epidermal cell

polymerase chain reaction

RPL resident pulmonary lymphocytes

\section{References}

$1 \mathrm{Sim}$, G. K. and Augustin, A. 1990. Dominantly inherited expression of BID, an invariant undiversified T cell receptor $\delta$ chain. Cell 61:397.

$2 \mathrm{Sim}$, G. K. and Augustin, A. 1991. Extrathymic positive selection of $\gamma \delta$ T cells: $V_{\gamma} 4 J_{\gamma} 1$ rearrangements with GXYS junctions. J. Immunol. 146:2439.

3 Augustin, A., Kubo, R. T. and Sim, G. K. 1989. Resident pulmonary lymphocytes expressing the $\gamma \delta T$ cell receptor. Nature 340:239.

4 Matis, L. A., Fry, A. M., Cron, R. Q., Cotterman, M. M., Dick, R. F. and Bluestone, J. A. 1989. Structure and specificity of a class II MHC alloreactive gamma delta $T$ cell receptor heterodimer. Science 245:746.

5 Bandeira, A., Itohara, S., Bonneville, M., Burlen-Defranoux, O., Mota-Santos, T., Coutinho, A. and Tonegawa, S. 1991. Extrathymic origin of intestinal intraepithelial lymphocytes bearing $T$-cell antigen receptor gamma delta. Proc. Natl Acad. Sci. USA 88:43.

6 Lefrancois, L., LeCorre, R., Mayo, J., Bluestone, J. A. and Goodman, T. 1990. Extrathymic selection of TCR gamma/delta ${ }^{+} T$ cells by class II major histocompatibility complex molecules. Cell 63:333.

7 Carding, S. R., Kyes, S., Jenkinson, E. J., Kingston, R., Bottomly, K., Owen, J. J. and Hayday, A. C. 1990. Developmentally regulated fetal thymic and extrathymic T-cell receptor gamma delta gene expression. Genes Dev. 4:1304.

8 Chomczynski, P. and Sacchi, N. 1987. Single step method of RNA isolation by acid guanidinium thiocyanate phenol chloroform extraction. Anal. Biochem. 162:156.

9 Rajasekar, R., Sim, G. K. and Augustin, A. 1990. Self heat shock and $\gamma \delta$ T cell reactivity. Proc. Natl Acad. Sci. USA 87:1767.

10 Chun, J. J., Schatz, D. G., Oettinger, M. A., Jaenisch, R. and Baltimore, D. 1991. The recombination activating gene-1 (RAG-1) transcript is present in the murine central nervous system. Cell 64:189.

11 Chelly, J., Kaplan, J. C., Maire, P., Gautron, S. and Kahn, A. 1988. Transcription of the dystrophin gene in human muscle and non-muscle tissue. Nature 333:858.

12 Dent, A. L., Matis, L. A., Hooshmand, F., Widacki, S. M., Bluestone, J. A. and Hedrick, S. M. 1990. Selt-reactive gamma delta $T$ cells are eliminated in the thymus. Nature 343:714.

13 Ito, K., Bonneville, M., Takagaki, Y., Nakanishi, N., Kanagawa, O.,
Krecko, E. G. and Tonegawa, S. 1989. T-cell receptors are expressed on thymocytes at different stages of development. Proc. Natl Acad. Sci. USA 86:631.

14 Allison, J. P. and Havran, W. L. 1991. The immunobiology of T cells with invariant gamma delta antigen receptors. Annu. Rev. Immunol. 9:679.

$15 \mathrm{Sim}, \mathrm{G}$. K. and Augustin, A. 1991. Dominant expression of the T cell receptor BALB invariant $\delta$ (BID) chain is due to selection. Eur. J. Immunol. 21:859.

16 Ikuta, K., Kina, T., MacNeil, I., Uchida, N., Peault, B., Chien, Y. and Weissman, I. L. 1990. A developmental switch in thymic lymphocyte maturation potential occurs at the level of hematopoietic stem cells. Cell 62:863

17 Turka, L. A., Schatz, D. G., Oettinger, M. A., Chun, J. J., Gorka, C. Lee, K., McCormack, W. T. and Thompson, C. B. 1991. Thymocyte expression of RAG-1 and RAG-2: termination by receptor cross-linking Science 253:778.

18 Borgulya, P., Kishi, H., Uematsu, Y. and von Boehmer, H. 1992 Exclusion and inclusion of $\alpha$ and $\beta$ T cell receptor alleles. Cell 69:529.

19 Sprent, J., Schaefer, M., Hurd, M., Surh, C. D. and Ron, Y. 1991. Mature murine $B$ and $T$ cells transferred to $S C I D$ mice can survive indefinitely and many maintain a virgin phenotype. J. Exp. Med. 174:717.

20 Rocha, B., Vassalli, P. and Guy-Grand, D. 1991. The V beta repertoire of mouse gut homodimeric alpha $\mathrm{CD}^{+}$intraepithelial $T$ cell receptor alpha/beta + lymphocytes reveals a major extrathymic pathway of $T$ cell differentiation. J. Exp. Med. 173:483

21 Poussier, P., Edouard, P., Lee, C., Binnie, M. and Julius, M. 1992. Thymus independent development and negative selection of $T$ cells expressing $T$ cell receptor $\alpha \beta$ in the intestinal epithelium: evidence for distinct circulation patterns of gut and thymus derived $T$ lymphocytes. J. Exp. Med. 176:187.

22 Goldman, J. P., Spencer, D. M. and Raulet, D. 1993. Ordered rearrangement of variable region genes of the T cell receptor $\gamma$ locus correlates with transcription of the unrearranged genes. J. Exp. Med. 177:729.

23 Asarnow, D. M., Cado, D. and Raulet, D. 1993. Selection is not required to produce invariant $T$ cell receptor $\gamma$ gene junctional sequences. Nature 362:158.

24 Elbe, A., Kilgu, O., Strohol, R., Payer, E., Schreiber, S. and Stingl, G. 1992. Fetal skin: a site for dendritic epidermal $T$ cell development. J. Immunol. 149:1694.

25 Mosley, R. L. and Klein, J. R. 1992. Peripheral engraftment of fetal intestine into athymic mice sponsors $T$ cell development: direc evidence for thymopoietic function of murine small intestine. J. Exp. Med. 176:1365.

26 Davodeau, F., Peyrat, M. A., Houde, I., Hallet, M. M.; De Libero, G., Vie, H. and Bonneville, M. 1993. Surface expression of two distinci functional antigen receptors on human gamma delta T cells. 260:1800

27 Padovan, E., Casorati, G., Dellabona, P., Meyer, S., Brockhaus, M. and Lanzavecchia, A. 1993. Expression of two T cell receptor alpha chains: dual receptor T cells. Science 262:422 